\title{
Ranas dardo venenosas (Dendrobatidae) y su importancia en la bioprospección de moléculas bioactivas en los últimos tiempos: una revisión
}

\section{Poison dart frog (Dendrobatidae) and their relevance in the bioprospection of bioactive molecules in recent times: a review}

\author{
Karol Gutiérrez ${ }^{1}$, Roger Morales ${ }^{2}$,Jorge Pino ${ }^{3 *}$ \\ ${ }^{1}$ Licenciatura en Química - Facultad de Ciencias Naturales y Exactas - Universidad Autónoma de Chiriquí, \\ ${ }^{2}$ Licenciatura en Biología Integral - Facultad de Ciencias Naturales y Exactas - Universidad Autónoma de Chiriquí \\ ${ }^{3}$ Vicerrectoría de Investigación y Postgrado - Universidad Autónoma de Chiriquí
}

\begin{abstract}
Resumen En esta revisión buscamos resaltar la importancia y la necesidad que tenemos por conocer más sobre lo que nos ofrece la naturaleza. Lograr comprender como influye distintos factores ecológicos sobre la supervivencia de los dendrobátidos, lo que ha logrado la evolución de la defensa química, los vacíos en el conocimiento de las ranas no aposemáticas, en su nivel de toxicidad. Enfocándonos principalmente en la importancia que ha tenido la familia Dendrobatidae, a nivel nacional e internacional en la farmacocinética, mediante la bioprospección de moléculas bioactivas, en los últimos tiempos. Ya que los estudios que se han realizados actualmente con los alcaloides, se ha descubierto que pueden ser modificados estructuralmente de forma fácil y económica. A nivel internacional, este tipo de investigaciones han tenido un gran auge. Panamá cuenta con 17 especies dentro de la familia Dendrobatidae, esto es importante ya que pueden ser especies con una fuente potencial de moléculas biológicamente activas y de esta manera se puede contribuir al desarrollo y fabricación de nuevos medicamentos.
\end{abstract}

Palabras claves Alcaloides, bioprospección, dendrobatidae, ecología química, moléculas bioactivas.

\begin{abstract}
In this review we seek to highlight the importance and the need that we have to know more about what nature offers us. To achieve an understanding of how different ecological factors, influence the survival of the dendrobatidae family, which achieved the evolution of chemical defense in them, the gaps in the ignorance of non-aposematic frogs in their level of toxicity. Focusing mainly on the importance of the dendrobatidae family, nationally and internationally in pharmacokinetics, through the bioprospecting of bioactive molecules, in recent times. Since current studies with alkaloids have found that they can be structurally modified easily and economically. At the international level, this type of research has been booming. Panama, has 17 species within the Dendrobatidae family, this is important as they can be species with a potential source of biologically active molecules and in this way can contribute to the development and manufacture of new drugs.
\end{abstract}

Keywords Alkaloids, bioprospecting, dendrobatidae, chemical ecology, bioactive molecules.

* Corresponding author: jorge.pino@unachi.ac.pa

\section{Introducción}

Compuestos químicos de productos naturales han sido utilizados desde tiempos muy remotos, su importancia, han surgido por el hecho de tener propiedades beneficiosas para el ser humano. Pero existen un grupo de compuestos químicos, que se les considera tóxicos o venenosos. Los mismos son utilizados en la naturaleza, ya sea por plantas o animales, como defensa para evitar ser depredados. Estos tipos de compuestos, han sido y seguirán siendo esenciales para la vida del ser humano. Mayormente en el caso de las plantas han sido utilizadas como fuente medicinal. En el caso de los animales, particularmente los anfibios han sido utilizados por tribus para la casa y otras lo han utilizado para rituales de curación (Daly, 1998).

Las ranas dardo venenosas, fueron estudiadas por el químico orgánico Jhon W. Daly, desde los años 60, las describe como especies que sobresalen por que presentan dentro de su arsenal químico compuestos biológicamente activos, los cuales son excretados por glándulas granulares 
que se encuentra en la parte lateral y superficial de la piel. El mismo se dedicó a identificar los compuestos que le daban el nombre ranas dardo venenosas, anteriormente nombradas por tribus indígenas, ya que envenenaban sus flechas para cazar. Jhon W. Daly, durante sus años de investigación con estos tipos de ranas, se encontró con una diversidad de compuestos biológicamente activos, importantes para el mundo de la ciencia (Saporito, 2012).

En la actualidad existen más de 850 alcaloides lipófilicos, que han sido aislados de la piel de estos anfibios. Entre estos alcaloides se encuentran: batracotoxina, que fue la primera en ser aislada y elucidada, histrionocotoxinas, pumilotoxinas, y epibatidina de las ranas venenosas del Neotrópico y muchos otros (Daly et al, 2005). Los alcaloides encontrados en este tipo de ranas son secuestrados de la dieta (artrópodos), aunque en la actualidad se ha descubierto que puede sintetizar nuevos compuestos. La función que cumplen estos compuestos es de defensa química contra los depredadores y microorganismos (Saporito et al, 2004).

El interés que despierta estos tipos de compuestos, encontrados ya sea en las plantas o en este caso los anfibios, es buscar su función en la farmacocinética para aplicación terapéutica en humanos. Particularmente los extractos obtenidos de la piel de este tipo de ranas se destacan por contener moléculas bioactivas, que puede tener, como, por ejemplo, una función en el tratamiento del dolor en enfermedades como el cáncer (Pasteels, 1983). Por eso este tipo de investigaciones referentes a la bioprospección de moléculas bioactivas, son más interesantes para el mundo de la ciencia.

El presente artículo aporta información de estudios realizados, sobre temas de elucidación estructural de compuestos bioactivos, encontrados en las ranas dardo venenosas de la familia Dendrobatidae, dándole importancia al desarrollo que ha tenido este tema a nivel nacional e internacional, con enfoque en la bioprospección de moléculas bioactivas para el mundo de la farmacocinética.

\section{Ecología química de los dendrobatidos}

La familia Dendrobatidae es un grupo monofilético con alrededor de 308 especies en 18 géneros, con una distribución endémica en el neotropico (Santos et al., 2009). Muchas de las especies de los dendrobátidos, se encuentran en una amplia distribución altitudinal, pueden estar alrededor de 2000 msnm, presentes en diferente tipo de bosques, tienen actividad diurna tanto como nocturna, de habitats terrestres, muy estrechas a un sitio con fuente de agua (río, arroyo). Son una de las familias más atractivas, buscadas para el comercio ilegal de mascotas, presentan gran variación de morfos en la coloración, dentro de cada especie (Lötters et al., 2007). Mucho de nuestros dendrobátidos existente en américa central son tóxicos o desagradables, con patrón críptico o aposemático lo cual es una señal de advertencia ante el depredador, por su capacidad de generar o tener un arsenal químico (Vences et al., 2003). En su reproducción, presentan comportamientos distintos, la mayoría colocan sus pequeñas masas de huevos en fitoltemas u hojarasca (Summers et al., 2006). En el caso de los dendrobátidos, cumplen un papel de cuidado paternal, donde les llevan a los renacuajos, ubicados en la fitotelma, huevos no fertilizados, para su respectiva alimentación (Summers y McKeon 2004). Estudios recientes demuestran que los huevos no fertilizados contienen alcaloides, los cuales han sido suministrado por la madre, esto nos indica que su alimento va acompañado de un arsenal químico para la defensa de los renacuajos (Stynoski et al., 2014a, b). La evolución de los dendrobátidos, en su defensa química, ha sido a causa de la selección natural del medio ambiente a la supervivencia al pasar del tiempo.

La composición de la defensa química y la dieta están estrechamente relacionadas, se ha reflejado que la mayoría de los compuestos químicos, tiene origen de los artrópodos (hormigas, ácaros, milpiés). Esto nos abre un gran campo para la investigación sobre la ecología química, de cómo, en la cadena trófica se adquiere, modifica o sintetiza un compuesto obtenido, que puede ser de origen vegetal (Daly et al., 1999, Saporito et al., 2012). Existen muchos vacíos en conocer con respecto al origen del clado, en el cual se inició la defensa química, muchos estudios se pueden realizar para conocer más sobre la composición química de aquellos dendrobátidos no aposemáticos. Mayormente, los estudios han sido enfocados en los dendrobátidos aposemáticos, por su gran distinción en presentar colores llamativos (Saporito et al 2009).

El aposematismo (Ruxton et al., 2004), de las especies se ha considerado como el síndrome fenotípico, el cual implica rasgos con variantes tales como: tamaño del cuerpo, la especialización de la dieta, el comportamiento que adopta frente situaciones de depredación y coloraciones. Esto ha modificado el comportamiento de búsqueda de predadores, promoviendo la asociación entre colores, olores o ciertos comportamientos y la incompatibilidad de la presa (Tullberg \& Hunter, 1996). Estas relaciones, se consideran como coevolución que puede ser obtenidas por selección natural o deriva genética (Pigliucci \& Preston, 2004). Esto ha hecho que las especies que adquieren estas modificaciones evolutivas, les permita acceder a los recursos que anteriormente no podían acceder, promoviendo un cambio representativo en la historia de su vida. (Lindquist \& Hay, 1996; Speed et al., 2010). Estas evoluciones pueden ser o no beneficiosas para los individuos, en el caso particularmente de los anfibios (ranas dardo venenosas). En otras especies aposemáticas como Dendrobates auratus, se presentan rangos 
Gutiérrez (et al): Ranas dardo venenosas (Dendrobatidae) y su importancia en la bioprospección de moléculas bioactivas en los últimos tiempos: una revisión

domésticos mayores y las crípticas (colores oscuros) presentan menor oportunidad de defensa contra sus predadores en su ambiente natural (Endler, 1988; van Wijngaarden, 1990). Por lo contrario, las especies crípticas presenta un mayor nivel de toxicidad que las especies de colores llamativos (Hernández, 2011). Se ha descubierto que existen correlaciones negativas y positivas entre la conspicuidad y la toxicidad de ciertas especies, como por ejemplo, se ha encontrado una correlación inversa entre conspicuidad y toxicidad en Oophaga granulifera y Oophaga pumilio, pero comparando ranas de sólo tres y dos localidades respectivamente (Daly \& Myers, 1967; Wang, 2011). El patrón opuesto se encontró al comparar las ranas O. pumilio de diez localidades en Panamá (Maan \& Cummings, 2012). Para entender más sobre estos resultados, se debe relacionar la conspicuidad, la toxicidad y la composición química del veneno, para poder entender si la evolución que ha tenido ha sido a nivel metabólico, pudiendo sintetizar nuevos compuestos biológicamente que los pueda hacer más tóxicos. Esto hace que este tipo de especies, se vuelvan más interesantes para estudios y saber su evolución en todos los sentidos.

\section{Primeros pasos sobre el conocimiento de los alcaloides lipófilicos en la familia Dendrobatidae}

Las tribus del Chocó, Emberá y Noanamá, fueron unas de las primeras en notar la toxicidad de este tipo de especies, ya que utilizaban los extractos de la piel para envenenar sus armas y poder cazar (Cochrane 1825; Posada-Arango 1883; Wassen 1935; Marki y Witkop 1963). Esto despertó interés en muchos investigadores bioquímicos, químicos orgánicos, entre otros. Los primeros en realizar estudios referentes a la función biológica del veneno de esta familia fueron Jhon W. Daly y Myers en 1967 (Jhon Daly, Myers, 1967). Luego el químico orgánico, Jhon W. Daly, en los más de 40 años de investigación que realizó, de esta familia, se enfocó en elucidar estructuras y buscar su función biológica. Los primeros estudios realizados por Jhon W. Daly en Panamá, fueron con la Oophaga pumillio en el archipiélago de Bocas del Toro.

La batracotoxina fue el primer alcaloide identificado en los extractos de la piel de las ranas venenosas, los cuales fueron identificados y aislados de tres especies del género Phyllobates (Dendrobatidae) encontradas en Colombia (Saporito et al., 2009).

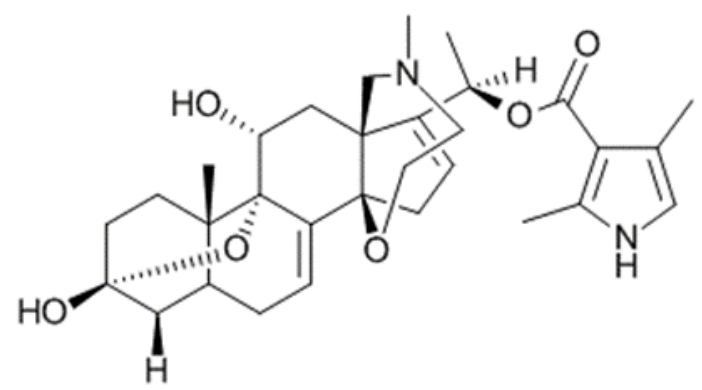

Figura 1. Primer alcaloide propuesto por el químico Jhon W. Daly. Estructura química de la Batrachotoxina.

\begin{tabular}{|c|c|}
\hline Pumiliotoxins & Izidines \\
\hline Pumiliotoxins (30) & 3,5-pyrrolizidines (26) \\
\hline Allopumiliotoxins (20) & 3,5-indolizidines (30) \\
\hline Homopumiliotoxins (18) & 5,8-indolizidines (80) \\
\hline \multirow{4}{*}{$\begin{array}{c}\text { Dehydropumiliotoxins } \\
\text { Desmethylpimiliotoxins(28) } \\
\text { Deoxypumiliotoxins }\end{array}$} & Dehydro5,8indolizidine(40) \\
\hline & 5,6,8-indolizidines (70) \\
\hline & 4,6-quinolizidines (6) \\
\hline & 1,4-quinolizidenes (20) \\
\hline$\underline{\text { Spiro-alakaloids }}$ & Ichmizidines (10) \\
\hline Histrionicotoxins (16) & Hydroxyzidines (25) \\
\hline \multicolumn{2}{|l|}{ Spiropyrrolizidines(8) } \\
\hline & Amides \\
\hline Decahydroquinolines & Epiquinamide (1) \\
\hline \multicolumn{2}{|l|}{ Decahydroquinolines (50) } \\
\hline \multirow[t]{2}{*}{ Dimers (7) } & Monocyclics \\
\hline & 2,5-pyrrolidines (9) \\
\hline$\underline{\text { Pyridinic alkaloids }}$ & 2,6-piperidines (40) \\
\hline \multicolumn{2}{|l|}{ Epibatidines (3) } \\
\hline \multirow[t]{2}{*}{ Other pyridinic alkaloids(4) } & Tricyclies \\
\hline & Gephyrotoxins (2) \\
\hline$\underline{\text { Steroidal alkaloids }}$ & Coccinelline-like (55) \\
\hline \multirow[t]{2}{*}{ Batrachotoxins (6) } & Cyclopentaquinolizidin(10) \\
\hline & Pseudophrynamines (13) \\
\hline \multicolumn{2}{|l|}{ Indolic alkaloids (2) } \\
\hline & Other alkaloids (150) \\
\hline
\end{tabular}

Figura 2. Número de alcaloides por cada clase, encontrados en los extractos de la piel. Se han encontrado más de 850 alcaloides, en los extractos de la piel de la familia Dendrobatidae.

En la actualidad existen más de 850 alcaloides descubiertos en los extractos de la piel de esta familia de anfibios Dendrobatidae. La mayoría de las estructuras encontradas en la piel de las ranas, pertenecen a la dieta (artrópodos que poseen alcaloides). Pero se ha descubierto que ellas pueden llegar a sintetizar compuestos nuevos derivados de los originales obtenidos en su dieta. Esto es para el caso de la alloPTX 251D, la cual fue sintetizada mediante 
una hidroxilación. Se sospecha que el mecanismo fue dado por Citocromo P450.

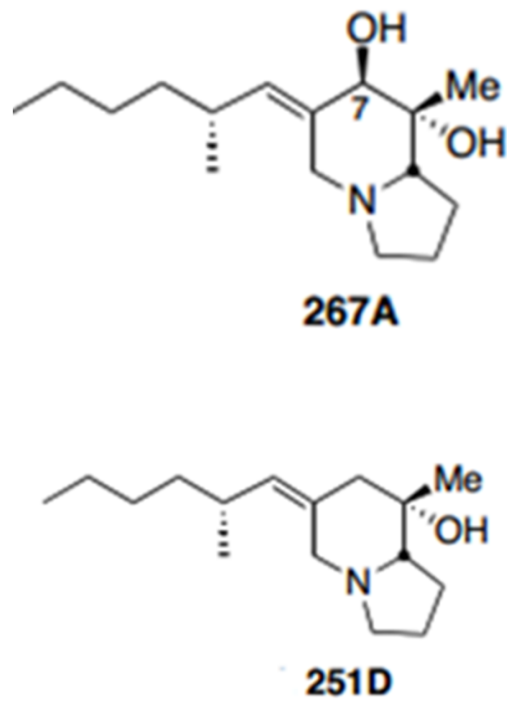

Figura 3. Estructura a de la pumiliotoxina 267A, secuestrada de la dieta Estructura b modificada por la especie.

\section{Importancia de la bioprospección de moléculas en el último siglo}

En la actualidad se ha estudiado bien a esta familia de ranas dardo venenosas, ya que presentan un sistema de evolución muy representativo, con respecto a sus estrategias defensiva, utilizando compuestos químicos secuestrados de la dieta para generar un arsenal químico contra sus depredadores. Las ranas dardo venenosas han tenido la capacidad de modificar moléculas, después de ser ingerida a partir de su dieta y gracias a las investigaciones de John W. Daly y sus colaboradores podemos saber las estructuras y sus funciones biológicas (Daly, 1994a).

Esto ha generado curiosidad, el hecho de conocer más sobre la función que pueda tener este tipo de especies, en el mundo de la farmacocinética. Estudios diversos han realizado, ya sea con alcaloides, aminas biogénicas o péptidos antimicrobianos, enfocándose en técnicas alternativas para el cáncer y resistencia bacteriana. Pero muchas más investigaciones se han hecho para seguir buscando el tratamiento de otras enfermedades. Con la ayuda de la biotecnología se podrán seguir desarrollando estos tipos de estudios buscando encontrar propiedades farmacológicoterapéutico a partir del arsenal químico encontrado en estos anfibios. Una propiedad muy importante se le ha encontrado a un tipo de alcaloide: la PTX (Pumiliotoxina), ya que es tóxico o repelente para el Aedes aegypti (Weldom, 2006).
Dentro del arsenal químico, encontrado en las ranas dardo venenosas, se encuentran compuestos biológicamente activos, pero los más potentes dentro de estos, son los alcaloides, ya que ha concentraciones más bajas tienen efecto. Los cuales lo hacen ser potenciales para el estudio a nivel farmacológico en diferentes organismos. En nuestro país el desarrollo que ha tenido este tipo de estudios con estas especies (ranas venenosas) se ha visto limitado, a pesar de la biodiversidad que presenta. Otros países a nivel de América, consideran que estas especies representan una manera de contribuir al desarrollo y fabricación de nuevos medicamentos.

El auge que ha tenido este tipo de estudios, ha sido grande, debido a que los alcaloides tienen grupos funcionales que los hacen capaces de ser modificados estructuralmente de forma fácil y económica. Con esto se propone evaluar más sobre este tema, ya que es completamente viable en el mundo de la farmacología, proponiendo a este tipo de ranas venenosas con fuente potencial de moléculas biológicamente activas.

\section{Conclusión}

La importancia en la evolución, del conocimiento de la familia Dendrobatidae, ha sido importante en el mundo de la ciencia, aunque existan vacíos por seguir completando, en las interrogantes que tenemos sobre esta familia, en la evolución que ha tenido por mucho tiempo, su importancia, en lo que nos brinda seguirá siendo esencial para el avance en el mundo de la bioprospección de moléculas bioactivas en la creación de fármacos para el tratamiento terapéutico de humanos.

El desarrollo que ha tenido este tema a nivel internacional ha sido un auge total. La mayoría de los artículos encontrados han sido presentados por investigadores internacionales de diferentes áreas de las ciencias. Panamá, ha sido objeto de estudio de estas personas, ya que nuestro país presenta una gran biodiversidad de especies de esta familia y es punto clave para investigaciones futuras sobre temas en la fabricación de fármacos.

\section{AGRADECIMIENTO}

Agradecemos a Dios todopoderoso por permitirnos contar con la sabiduría, fuerza, entendimiento y la paciencia con la que debíamos contar para poder llevar a cabo con éxito este proyecto de revisión. 


\section{REFERENCIAS}

[1] J.W. Daly, "Thirty years of discovering arthropod alkaloids in amphibian skin". J Nat Prod 61:162-172, (1998).

[2] R.A. Saporito, Donnelly MA, Spande TF, Garraffo HM A, "Review of chemical ecology in poison frogs". Chemoecology 22:159-168, (2012).

[3] J.W. Daly, T.F. Spande, H.M. Garraffo, "Alkaloids from amphibian skin: a tabulation of over eight-hundred compounds". J Nat Prod 68:1556-157, (2005).

[4] R.A. Saporito, H.M. Garraffo, M.A. Donnelly, A.L. Edwards, J.T. Longino, J.W. Daly, "Formicine ants: an arthropod source for the pumiliotoxin alkaloids of dendrobatid poison frogs". PNAS 101:8045-8050, (2004).

[5] J.M. Pasteels, J-C. Gregoire, M. Rowell-Rahier, "The chemical ecology of defense in arthropods". Annu Rev Entomol 28:263-289, (1983).

[6] J.C. Santos, L.A. Coloma, K. Summers, J.P. Caldwell, R .Ree, D.C. Cannatella, "Amazonian amphibian diversity is primarily derived from late Miocene Andean lineages". (2009).

[7] S. Lötters, K.H. Jungfer, F.W. Henkel, W. Schmidt, "Poison frogs. Biology, species and captive husbandry". Edition Chimaira, Frankfurt am Main, (2007).

[8] M. Vences, J. Kosuch, R. Boistel, C.F.B. Haddad, La Marca E, Lötters S, Veith M, "Convergent evolution of aposematic coloration in neotropical poison frogs: a molecular phylogenetic perspective". Organ Div Evol 3:215-226, (2003).

[9] K. Summers, C.S. McKeon, H. Heying, "The evolution of parental care and egg size: a comparative analysis in frogs". Proc R Soc B Biol Sci 273:687-692, (2006).

[10] K. Summers, C.S. McKeon, "The evolutionary ecology of phytotelmata use in neotropical poison frogs". Misc Publ Univ Mich Mus Zool 193:55-73, (2004).

[11] J.L. Stynoski, Y. Torres-Mendoza, M. Sasa-Marin, R.A. Saporito, "Evidence of maternal provisioning of alkaloidbased chemical defenses in the strawberry poison frog Oophaga pumilio. Ecology 95:587-593, (2014a).

[12] J.L. Stynoski, G. Shelton, P. Stynoski, "Maternally derived chemical defences are an effective deterrent against some predators of poison frog tadpoles (Oophaga pumilio)". Biol Lett 10:4, (2014b)

[13] J.W. Daly, H.M. Garraffo, T.F. Spande, "Alkaloids from amphibian skins. In: Pelletier SW (ed) Alkaloids: chemical and biological perspectives". Pergamon Press, New York, pp 1-161, (1999).

[14] R.A. Saporito, T.F. Spande, H.M. Garraffo, M.A. Donnelly, "Arthropod alkaloids in poison frogs: a review of the dietary hypothesis". Heterocycles 79:277-29, (2009).

[15] G. Ruxton, T. Sherrat., \& M. P. Speed. "The form and function of warning signals Avoiding Attack: The Evolutionary Ecology of Crypsys, Warning Signals and Mimicry" (pp. 82-101). New York: Oxford Univ. Press, (2004).

[16] B. Tullberg, \& A. Hunter, "Evolution of larval gregariousness in relation to repellent defenses and warning coloration in tree-feeding Macrolepidoptera: A phylogenetic analysis based on independent contrasts”. Biol. J. Linn. Soc. Lond., 57, 253-276, (1996).

[17] M. Pigliucci, \& K. Preston, "The Diversity of Complexity Phenotypic Integration: Studying the Ecology and Evolution of Complex Phenotypes" (pp. 3-20). New York: Oxford Univ. Press, (2004).

[18] N. Lindquist, \& M. Hay, "Palatability and chemical defense of marine invertebrate larvae". Ecol. Monogr, 66, 431-450, (1996).

[19] M.P. Speed, M. A. Brockhurst, \& G. D. Ruxton, "The dual benefits of aposematism: predator avoidance and enhanced resource collection”. Evolution, 64 (6), 1622-1633, (2010)

[20] J. Endler, "Frequency-dependent predation, crypsis and aposematic coloration”. Lond. Ser. Biol. Sci., 319, 502-523, (1988).

[21] Hernández, "Beauty does not always denote danger: Aposematic syndrome in the poison frog Dendrobates auratus". Universidad de los Andes, Facultad de Ciencias, Departamento de Ciencias Biológicas, Grupo de Ecofisiología Comportamiento y Herpetología, (2011).

[22] J.W. Daly, C.W. Myers, "Toxicity of Panamanian poison frogs (Dendrobates): some biological and chemical aspects". Science 156:970-973, (1967).

[23] I. J. Wang, "Inversely related aposematic traits: reduced conspicuousness evolves with increased toxicity in a polymorphic poison-dart frog”. Evolution, 65 (6), 16371649, (2011).

[24] M. E. Maan, \& M. E. Cummings, "Poison frog colors are honest signals of toxicity, particularly for bird predators". Am. Nat., 179 (1), E1 -E14, (2012).

[25] C.S. Cochrane, "Journal of a residence and travels in Colombia, during the years 1823 and 1824". Printed for Henry Colburn, London, (1825).

[26] A. Posada-Arango, "Veneno de la rana. Usado por los salvajes del Chocó". Revista Médica de Bogota 7:451-456. (1883).

[27] S.H. Wassen, "Notes on southern groups of Chocó Indians in Colombia". Ethnografi ska Museet. Göteborg, Etnologiska Studier 1:35-182, (1935).

[28] F. Marki, B. Witkop, "The venom of the Colombian arrow poison frog Phyllobates bicolor". Experientia 19:329-376, (1963).

[29] J.W. Daly, H.M. Garraffo, C. Jaramillo, A.S. Rand, "Dietary source for skin alkaloids of poison frogs" (Dendrobatidae) J Chem Ecol 20:943-95, (1994a).

[30] P.J. Weldon, M. Kramer, S. Gordon, T.F. Spande, J.W. Daly, "A common pumiliotoxin from poison frogs exhibits enantioselective toxicity against mosquitoes". PNAS 103:17818-1782, (2006). 